\title{
Iliac Artery-Uretero-Colonic Fistula Presenting as Gastrointestinal Hemorrhage and Hematuria: A Case Report
}

\author{
Saya Kurata, MD, ${ }^{1,2}$ Shohei Tobu, MD, ${ }^{2}$ Kazuma Udo, MD, and Mitsuru Noguchi, $\mathrm{MD}^{2}$
}

\begin{abstract}
Background: The experience with uretero-arterial fistulas has been limited. However, the aggressive treatment of pelvic tumors with surgical resection and radiotherapy, along with liberal use of ureteral catheters, has been attributed to an increase in their incidence. Unless they are promptly diagnosed and treated, uretero-arterial fistulas are associated with considerably high rates of morbidity and mortality. Urologists need maintain a high degree of suspicion for uretero-arterial fistula in high-risk patients. We herein present the clinical course of an iliac artery-uretero-colonic fistula.

Case Presentation: A 67-year-old woman with a history of colon cancer who underwent laparoscopic high anterior resection in July 2010. A ureteral stent inserted to right ureteral stricture, which developed as a result of local recurrence of the tumor in September 2010. She had undergone chemoradiotherapy, but the lesion had slowly increased in size. During the replacement of the ureteral stent in April 2016, she immediately experienced bladder tamponade, bloody bowel discharge, and hypotension. Contrast CT revealed a complex fistula between the right distal ureter and the right internal iliac artery. Furthermore, contrast medium flowed into the intestinal tract through the tumor. The patient was therefore diagnosed with internal iliac artery-uretero-colonic fistula. Arteriography revealed a right uretero-internal iliac artery fistula, and the embolization of the right internal iliac artery was performed. The right ureteral stent was removed. Her hematuria and bloody bowel discharge disappeared, but right nephrostomy was performed because she presented with acute pyelonephritis to ureteral obstruction.

Conclusion: In the present case, the uretero-arterial fistula was caused by the long use of an indwelling stent, chemoradiotherapy, infection, and an increase in the size of the lesion. When a suspected uretero-arterial fistula is accompanied by bloody bowel discharge, we should consider the possibility of traffic to the intestinal tract.
\end{abstract}

Keywords: ureteral stricture, uretero-arterial fistula, arterioenteric fistula, artery embolization

\section{Introduction and Background}

$\mathbf{U}$ ROLOGISTS PERFORM URETERAL stent placement to improve hydronephrosis in patients with nonurologic primary malignancies; the obstruction occurs due to direct invasion, nodal disease, or inflammation. Because urologists have many opportunities to insert ureteral stents, it is necessary to have a thorough understanding of the potential complications. We herein report the case of an iliac arteryuretero-colonic fistula in a patient who had been managed by ureteral stenting for 6 years due to severe ureteral stricture, which developed after laparoscopic high anterior resection with pelvic irradiation and chemotherapy for advanced colon cancer.

\section{The Case Presentation}

The patient was a 67-year-old woman with a history of colon cancer who had undergone laparoscopic high anterior resection in July 2010. She presented with local recurrence at the pelvic surface of the sacrum in September 2010. The lesion involved the right ureter and caused right

\footnotetext{
${ }^{1}$ Department of Urology, Nagasaki Kawatana Medical Center, Nagasaki, Japan.

${ }^{2}$ Department of Urology, Faculty of Medicine, Saga University, Saga, Japan.
}

(c) Saya Kurata et al. 2018; Published by Mary Ann Liebert, Inc. This is an Open Access article distributed under the terms of the Creative Commons Attribution License, which permits unrestricted use, distribution, and reproduction in any medium, provided the original work is properly cited. 
FIG. 1. Contrast $\mathrm{CT}$ at admission. (A) Clot in the right renal pelvis. (B) Recurrence
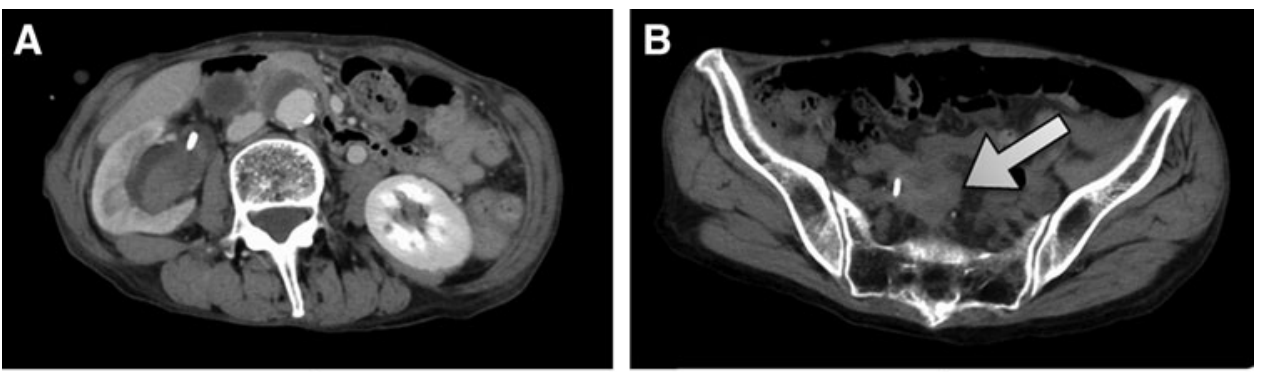
tumor (arrow). (C) Clot in the rectum.

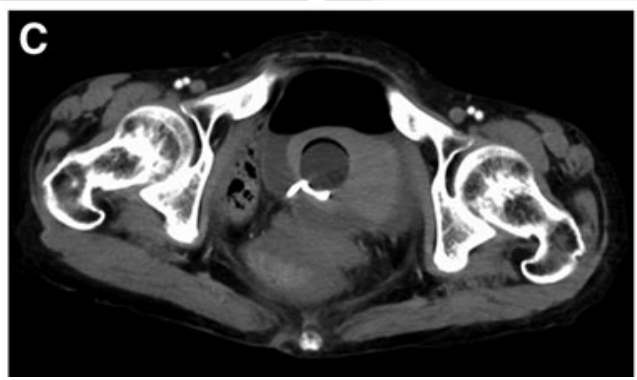

hydronephrosis. We inserted a ureteral stent due to right back pain and renal dysfunction. The ureteral stricture was slightly distal to the bifurcation of the common iliac artery. The stent was regularly changed every 3 months. However, acute pyelonephritis due to vesicoureteral reflux sometimes developed and she was treated with antibiotics. She continued to undergo chemotherapy; however, in February 2016, CT revealed that the tumor had increased in size.

In April 2016, the patient experienced hematuria after routine ureteral stent replacement. Three days later, she was urgently hospitalized for severe hematuria. The hematuria was intermittent but progressed to severe hematuria and lower gastrointestinal hemorrhaging over a short period of time. She required blood transfusions at every bleeding episode due to anemia.

We were unable to identify the site of bleeding by colonoscopy or contrast-enhanced CT (Fig. 1). A clot with hematuria extended from the ureter to the pelvis renalis, and the ureteral stent became occluded, causing the patient to de- velop acute pyelonephritis. Because there was no improvement after the administration of an antimicrobial agent, we decided to change the ureteral stent. After removing the stent, we attempted to insert a guidewire but felt strong resistance at the ureteral stricture. The urinary tract showed no contrast on retrograde pyelography (RP). We attempted several times to maneuver the guidewire past the stricture but were unsuccessful. After performing RP again, a vascular structure out of the urinary tract was contrasted. Subsequently, pulsatile bleeding occurred through the right ureteral stent. She immediately experienced bladder tamponade, lower gastrointestinal hemorrhage, and hypotension. We inserted a urethral catheter and a ureteral catheter, which was ligated to the urethral catheter to ensure that the position of the tip did not move. We then fixed it and clamped the exit portion of the catheter. Contrast CT revealed a complex fistula between the right distal ureter and the right internal iliac artery (Fig. 2). Contrast medium also flowed into the recurrent tumor, as well as into the intestinal tract through the tumor. Based on these
FIG. 2. Contrast $\mathrm{CT}$ at bleeding. (A) Contrast medium in the tumor. (B) Contrast medium in the ileum. (C) Contrast medium in the bladder.
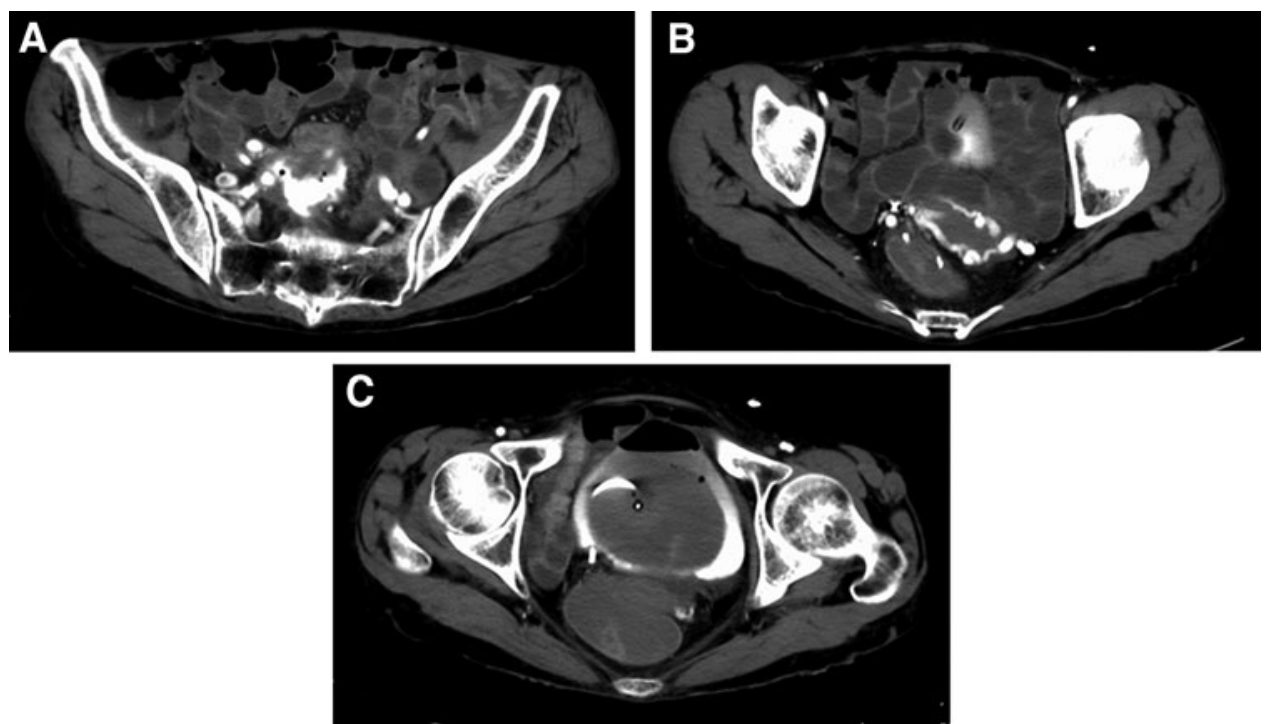

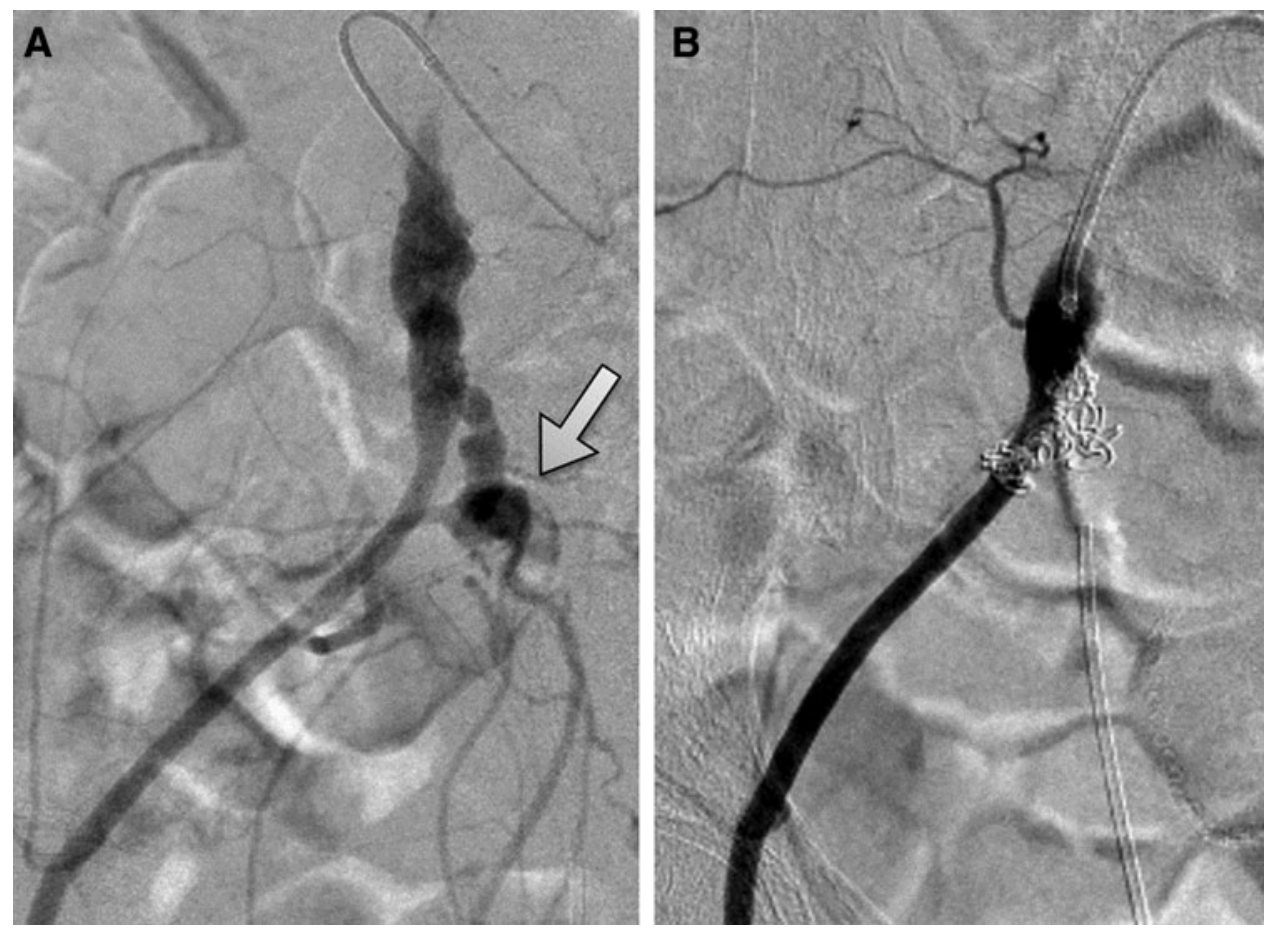

FIG. 3. Angiogram. (A) The right uretero-internal iliac artery fistula. (B) After embolization of the right internal iliac artery. findings, the patient was diagnosed with an internal iliac artery-uretero-colonic fistula. Arteriography revealed a right uretero-internal iliac artery fistula, and embolization of the right internal iliac artery was performed (Fig. 3). After embolization, the ureteral stent was carefully removed. Hematuria and bloody bowel discharge disappeared; however, right nephrostomy was performed because she presented with acute pyelonephritis due to ureteral obstruction. At present, she continues to receive chemotherapy and the periodic exchange of the right nephrostomy. There has been no relapse of hematuria.

\section{Discussion and Literature Review}

Uretero-arterial fistulas are uncommon; however, if they are not promptly diagnosed and treated, then these fistulas may be associated with considerably high rates of morbidity and mortality. Before 1980, the reported mortality rate was $69 \%$; however, this has dropped to $23 \%$ in recent years. ${ }^{1} \mathrm{An}$ increased awareness of uretero-arterial fistula and diagnostic techniques that can achieve a rapid diagnosis has improved patient outcomes. Fistulas can occur in association with several conditions, including prior pelvic surgery, pelvic radiation, chronic inflammatory conditions of the pelvis, the placement of indwelling ureteral stents, and primary vascular disease. Prior surgery or radiation can further lead to microvascular changes in the arterial vasa vasorum, which can lead to ischemic changes. These arteries are then more susceptible to rupture, erosion, and necrosis. ${ }^{2}$ If a ureteral stent is present, it can essentially act as a backboard onto which a pulsatile artery will repeatedly transmit a systolic pressure wave onto the interposing arterial and ureteral walls. Ureteral stent changes can further promote the process by causing microabrasion of the ureteral lumen. Over time a fistula can occur secondarily to pressure necrosis.
Fistulas commonly involve the ipsilateral common iliac artery or even the external or internal iliac artery. There are no consistent opinions on sex differences, laterality, or the frequency according to the site. The occurrence of ureteroarterial fistulas is increasing. The aggressive treatment of pelvic tumors with surgical resection and radiotherapy and the improvement of the survival rate, along with the liberal use of ureteral stents, have been attributed to this increase in occurrence.

In approximately half of all cases, uretero-arterial fistulas present with gross hematuria along with flank pain due to the obstruction of the ureter by blood clots. Bleeding can be massive and dramatic or intermittent in nature. The high rate of mortality associated with uretero-arterial fistulas has been attributed to delays in the diagnosis and treatment. A high index of suspicion is needed to make the diagnosis, not only because the hematuria may be intermittent in nature but also because demonstrating the diagnosis can be challenging. There is no optimum diagnostic modality, as no diagnostic methods are particularly sensitive for such fistulas; thus, a negative result on any study does not necessarily exclude the diagnosis of a uretero-arterial fistula. Although CT is commonly performed in the emergency room, this modality shows low sensitivity and is only diagnostic in $50 \%$ of cases. ${ }^{3}$ Cystoscopy may reveal lateralizing blood flow, which may be pulsatile. RP is often nondiagnostic. A single test is not usually diagnostic, and repeated radiologic examinations are required to establish the diagnosis. Angiography is even less sensitive $(23 \%-41 \%)^{3}$; however, provocative angiography should be performed when the indwelling ureteral stent is manipulated, and the angiographic catheters should be manipulated to allow for direct infusion over the suspected fistula site until bleeding is seen. This method was shown to improve the sensitivity to $100 \%$ in 1 series. ${ }^{3}$ However, because life-threatening bleeding can be provoked by the manipulation, it is necessary to perform this manipulation in 
cooperation with a multidisciplinary team. In the present case, the bleeding point was not initially known after the patient's bleeding stopped, and a clot was the only anomaly detected on cystography CT; however, a fistula was still able to be diagnosed by urgent cystography CT, RP, and angiography at the time of bleeding.

There are two main therapeutic approaches for ureteroarterial fistulas: open surgery and endovascular treatment. The open surgery procedures include nephrectomy, nephroureterectomy, bypass artery surgery, aneurysmectomy, and replacement with a vascular graft. However, open surgery is often tedious and risky due to preceding treatments such as pelvic surgery and pelvic irradiation. Endovascular treatment has now become an appealing alternative to open surgical reconstruction, as it is minimally invasive, which allows for an earlier patient recovery, and because it appears to be as effective as open surgery for controlling bleeding. Endovascular treatment is used increasingly frequently in lieu of open surgery due to the high operative risk associated with an open approach and the presence of comorbidities in patients with uretero-arterial fistulas. Endovascular treatment included stent grafting and embolization. The use of stent grafts is contraindicated in cases of infection.

The present case was complicated with uretero-arterial and arterioenteric fistulas. ${ }^{4}$ Arterioenteric fistulas typically occur following aortoiliac interventions for aneurysmal disease; however, primary fistula formation can be caused by an infectious process, malignancy, radiation exposure, or by the ingestion of a foreign body. ${ }^{4}$ Patients present with lower gastrointestinal bleeding, ranging from minor hematochezia to hemorrhagic shock. In $44 \%$ of subjects, the common iliac artery is involved in the arterioenteric fistula, whereas the internal iliac artery and external iliac artery are involved in $33 \%$ and $26 \%$ of cases, respectively. The affected bowel segment was the colon in $51 \%$ of patients, the small bowel in $31 \%$, and the rectum in $21 \%$. Patients who present with arterioenteric fistula often undergo a plethora of diagnostic studies, including upper and lower endoscopy, CT, angiography, and nuclear bleeding scans. Although appropriate, these are sometimes nondiagnostic. The treatments include open surgeries and endovascular therapy; the 30-day mortality rate is reported to be $26 \%$.

Iliac artery-uretero-colonic-fistula is extremely rare. However, the mechanism underlying the development of arterioenteric fistula is extremely similar to that of uretero-arterial fistula. When uretero-arterial fistula is suspected and severe hemorrhaging is present, the physician should assume that the gastric system is involved.

\section{Conclusion}

Uretero-arterial fistula is a rare complication that can occur in patients with long-term indwelling ureteral stent. Unless it is promptly diagnosed and adequately treated, the condition is potentially fatal. Heightened awareness and a high index of suspicion for this condition are required to make an early diagnosis. When bloody bowel discharge occurs in a patient with suspected uretero-arterial fistula, the possibility of traffic to intestinal tract should be considered.

\section{Disclosure Statement}

No competing financial interests exit.

\section{References}

1. Yamasaki K, Omori K, Takaoka E, et al. Successful management by provocative angiography and endovascular stent of Ureteroarterial fistula in a patient with a long-term indwelling ureteral stent. Jpn J Clin Oncol 2010;40:267-270.

2. Puppo P, Perachino M, Ricciotti G, Zinicola N, et al. Ureteroarterial fistula: A case report. J Urol 1992;148:863-864.

3. Krambeck AE, DiMarco DS, Gettman MT, Segura JW. Ureteroiliac artery fistula: Diagnosis and treatment algorithm. Urology 2005;66:990-994.

4. Policha A, Baldwin M, Mussa F, Rockman C. Iliac arteryuretero-colonic fistula presenting as severe gastrointestinal hemorrhage and hematuria: A case report and review of the literature. Ann Vasc Surg 2015;29:1656.e1-e6.

Address correspondence to:

Saya Kurata, MD

Department of Urology

Nagasaki Kawatana Medical Center

Shimokumigo 2005-1

Kawatana-town

Higasisonogi-gun

Nagasaki 859-3615

Japan

E-mail:wtantanda@gmail.com

Cite this article as: Kurata S, Tobu S, Udo K, Noguchi

M (2018) Iliac artery-uretero-colonic fistula presenting as gastrointestinal hemorrhage and hematuria: a case report, Journal of Endourology Case Reports 4:1, 1-4, DOI: $10.1089 /$ cren.2017.0066.

\footnotetext{
Abbreviations Used

$\mathrm{CT}=$ computed tomography

$\mathrm{RP}=$ retrograde pyelography
} 